\title{
Epidural anesthesia for the patient with type IV spinal muscular atrophy -A case report-
}

\author{
Seon Jin Kim, Eun Ju Kim, Byung Woo Min, Jong Seouk Ban, Sang Gon Lee, and Ji Hyang Lee \\ Department of Anesthesiology and Pain Medicine, Daegu Fatima Hospital, Daegu, Korea
}

Spinal muscular atrophy (SMA) is a rare lower motor neurone disease in which anesthetic management is often difficult as a result of muscle weakness, hypersensitivity to non-depolarizing neuromuscular blocking agent, and succinylcholine induced hyperkalemia. We describe a 50-year-old patient with type IV SMA for synovectomy. We decided to use an epidural technique to avoid muscle relaxants and tracheal intubation. After operation, there was no exacerbation of neurologic signs and symptoms. (Korean J Anesthesiol 2010; 59: S65-S68)

Key Words: Epidural anesthesia, Spinal muscular atrophy.

Spinal muscular atrophy (SMA) is a rare lower motor neuron disease that occurrs due to degeneration of anterior horn cell. SMA patients require particular attention for narcosis management since there is a possibility for mechanical ventilation when a delayed recovery of spontaneous respiration occurs after an operation using general anesthesia as a sensitive reaction against skeletal muscle relaxants and weakened respiratory root [1]. Accordingly, regional anesthesia can be an option as an alternative of general anesthesia for lower extremity surgery.

The authors successfully conducted narcosis management for epidural anesthesia for patients with type IV spinal muscular atrophy without worsening the neurological symptoms or creating respiratory complications. Since there has not been any previous domestic report about regional anesthesia for patients with spinal muscular atrophy, this study reports the case.

\section{Case Report}

A 50-year-old male patient visited the hospital to undergo synovectomy for left knee ostarthritis and tenosynovitis. The patient had suffered difficulties in walking from weakening of both lower limb muscles since 25 years ago, and recently the symptom of weakness of lower limb muscles became more serious and dyspnea at the level of ATS (American Thoracic Society) Dyspnea Grade I had occurred due to the weakening of the respiratory root. A molecular genetics examination conducted at another hospital discovered a deficit of the SMN1 gene, and considering the time of onset and clinical conditions, the patient was diagnosed with type IV spinal muscular atrophy.

Received: April 19, 2010. Revised: 1st, May 11, 2010; 2nd, June 6, 2010. Accepted: July 1, 2010.

Corresponding author: Eun Ju Kim, M.D., Department of Anesthesiology and Pain Medicine, Daegu Fatima Hospital, 302-1, Sinam-dong, Dong-gu, Daegu 701-010, Korea. Tel: 82-53-940-7434, Fax: 82-53-954-7417, E-mail: kej1127@fatima.or.kr

(c) This is an open-access article distributed under the terms of the Creative Commons Attribution Non-Commercial License (http:// creativecommons.org/licenses/by-nc/3.0/), which permits unrestricted non-commercial use, distribution, and reproduction in any medium, provided the original work is properly cited. 
The patient stood $168 \mathrm{~cm}$ and weighed $63 \mathrm{~kg}$, and there were no special opinions from ECG and simple radioactive examination before operation. Arterial blood gas analysis (ABGA) showed pH 7.435, $\mathrm{PaO}_{2} 74.6 \mathrm{mmHg}, \mathrm{PaCO}_{2} 36.9 \mathrm{mmHg}$, and $\mathrm{HCO}_{3}-24.2$ $\mathrm{mmHg}$. The patient was under drug treatment for hypertension. Administration before anesthesia was not conducted and vital signs examined after going into the operating room were blood pressure: 160/100 mmHg; respiration rate per minute: 20 times; ECG: normal sinus rhythm; and pulse oxymetry: $98 \%$. From a manual muscle test before operation, both legs were at the 4 th stage. At the genupectoral position, the epidural space was identified by loss of resistance technique using an 18 G Tuohy insertion needle in between the L2-L3 lumbar, and after a test dose injection of $1.5 \%$ lidocaine $3 \mathrm{ml}$ containing 1 : 200,000 epinephrine, no increase of heart rate or spinal anesthesia symptom was detected. After injecting $0.75 \%$ ropivacaine $12 \mathrm{ml}$, a catheter was placed and maintained. To measure the level of motor block by time after anesthesia, a modified Bromage scale (0: One can stretch and raise his/her leg; 1: One cannot raise his/her leg and cannot bend his/her knee; 2: One cannot his/ her knee but can bend his/her ankle; 3: One cannot bend his/ her ankle) was applied.

The level of modified Bromage scale before anesthesia was 1, and 20 minutes after injecting ropivacaine, the level of modified Bromage scale was 3 . The sensory nerves were blocked by T8 segment on pinprick examination. Vital signs during operation were blood pressure: $120-140 / 60-80 \mathrm{mmHg}$ and pulse rate per minute: stable at $70-80$ times. A Venti ${ }^{\circledR}$ mask with $35 \%$ of oxygen fraction in inspired air was used, and an $\mathrm{ETCO}_{2}$ capnometer was placed under the nose of the patient to monitor the end-tidal $\mathrm{CO}_{2}$. $\mathrm{ETCO}_{2}$ was stable at $35 \mathrm{mmHg}$ and the pulse oxymetry was $100 \%$. Due to the risk of aspiration of saliva or bronchial secretion during operation, sedation was not operated. The surgery was operated for an hour, and the epidural catheter was removed after operation. After finishing the operation, the level of modified Bromage scale was 3 and the sensory nerve block was decreased up to the T9 segment. Three hours after the operation, the level of modified Bromage scale was dropped to 2 and the sensory nerve block to the T11 segment; 5 hours after the operation, the stage of modified Bromage scale was 1 , and the sensory nerve was declined to the L1 segment. Since the patient did not show adverse effects including vomiting, nausea, and dizziness in the recovery room, he was moved to a patient room. Twelve hours after finishing the operation, both the level of the modified Bromage scale and sensory nerve were recovered to the condition before operation, and also for the manual muscle test, both legs were maintained at the 4th stage. A physical exam conducted 24 hours after finishing surgery did not show special opinions. The patient recovered smoothly without complications and left the hospital
2 days after the operation.

\section{Discussion}

Spinal muscular atrophy is a rare lower motor neuron disease, which does not accompany sensory loss, and is an autosomal recessive disorder that occurrs due to a genetic deficit of the survival motor neuron (SMN) existing in chromosome 5. The incidence rate is $1 / 10,000$ while it occurs as an autosomal recessive within $95-98 \%$ and an autosomal dominant within $2-5 \%$ [1]. The disease incurs motor nerve regression of the brain stem and anterior horn, leading to limb weakness and atrophy of the torso and proximal biceps tendon, paradoxical breathing related with bulbar paralysis, repeated upper respiratory infection, and severe kyphoscoliosis. Restrictive lung disease often occurs due to skeleton malformations, including kyphoscoliosis or contracture, and the patient cannot stand or walk according to his/her own will and sometimes rely on an artificial respirator [2].

For diagnosis of spinal muscular atrophy, clinical symptoms are most critical, and for differential diagnosis, serum enzymes measurements including $\mathrm{CK}, \mathrm{LDH}$, aldolase, aspartate aminotransferase (AST), alanine aminotransferase (ALT), electromyogram test, and muscle biopsy examination are useful. As the SMN gene and neuronal apoptosis inhibitory protein (NAPI) gene were discovered by molecular genetics research, a molecular genetics diagnosis became available [3]. Spinal muscular atrophy can be classified by a number of criteria including clinical conditions, genotype, and onset timing. Among them, the ISMAC (International SMA Consortium) system, which classifies the disease by onset timing, is applied the most widely. It divides the types of disease by type I: within 6 months after birth; type II: between 6 and 18 months; type III: after 18 month from birth; and type IV: for adults in the middle of one's 30s. Type I spinal muscular disease (WerdnigHoffman disease) occurs within 6 months from the date of birth, and most of the patients die within 18 months due to serious muscle weakness and myelencephalon hypofunction. Type II spinal muscular atrophy, the most common type, occurs between 6 and 18 months and most of the patients die within 2 years. The symptoms are milder hypotonus and fasciculation than type I. Type III spinal muscular atrophy (KugelbergWelander syndrome) occurs 18 months after the birth, and its characteristic is that symptoms are mild and proceed slowly. Most patients can have a normal lifestyle [1]. For adulthood disease, Pearn classified the type IV spinal muscular atrophy, and clinical conditions that mostly occur in the middle of a patient's 30s with mild symptoms, as type III [4]. Recovery after general anesthesia of patients with spinal muscular atrophy can be delayed because of restrictive lung disease 
from malformation and respiratory muscle weakness. Also, the most important thing in conducting general anesthesia for patients with spinal muscular atrophy is the use of skeletal muscle relaxants. Administration of skeletal muscle relaxants for patients with spinal muscular atrophy extends muscle relaxation and delays recovery, so special attention should be paid to their use.

Since acetylcholine is decreased from declined choline acetyltransferase from regression and atrophy of anterior horn cell, the sensitivity toward the depolarizing skeleton muscle relaxants increases and, at the same time, there is the possibility of respiratory root weakness and aspiration due to muscle regression. Against this backdrop, special attention should be paid to the use of skeleton muscle relaxants, dose setting, and reverse of the nerve block [5]. Also, succinylcholine is a taboo for the risk of extreme hyperkalemia and rhabdomyolysis. Accordingly, regional anesthesia can be an alternative to general anesthesia for bupivacaine. However, some worry that regional anesthesia for patients with spinal muscular atrophy can cause neurologic deficit, including worsening of limb motor nerve weakness. Upton and McComas [6] insist that if patients who have already suffered neurological malfunction are exposed to secondary damages such as injuries from needles or catheters, ischemic damages from vasopressors, or toxicity of a local anesthetic, the possibility for neurological damages increases. However, Hebl et al. [7] argue that neurological damages after operation occur from the combination of various factors including the lifestyle of patients, damages during operation, pressure of the tourniquet, posture of the patients during operation, dystocia, and anesthesia methods, and for diseases with progressive neurological damages, neurologic symptoms can be worsened regardless of methods of anesthesia and operations. Moreover, they said there was no case in which new or worsened neurologic damages occurred among 136 patients who had diseases showing neurologic dysfunction and who underwent regional anesthesia and pain control. Also, they added that worsening neurologic symptoms after regional anesthesia is not as common as expected in the past, and the operation of regional anesthesia is not taboo at all. Some patients with diseases in the central nervous system reported the case of worsening neurological function when operating regional anesthesia [8]; however, there was none for worsening neurological function after regional anesthesia for patients with a lower motor neuron disease. Moreover, operating regional anesthesia for patients with spinal muscular atrophy has advantages in that it can avoid possible respiratory complications or unanticipated extension of muscle relaxation. Weston and DiFazio [9] reported the case that conducted epidural pain control during delivery for a patient with type III spinal muscular atrophy, and Buettner [10] reported the case that conducted Cesarean delivery with epidural anesthesia for a patient with type III spinal muscular atrophy. Also, there is a report about the case that conducted regional anesthesia without neurological damages for patients with lower motor nervous disease including amyotrophic lateral sclerosis [11]. Domestically, there has been no case report yet about regional anesthesia for patients with spinal muscular atrophy. Meanwhile, Choi et al. [12] conducted total intravenous anesthesia without muscle relaxants for patients with type II spinal muscular atrophy, while Hong et al. [13] did for patients with type III spinal muscular atrophy. Both cases were for head and neck surgeries, and it is considered that general anesthesia was conducted for children patients. Consequently, conducting regional anesthesia for patients with neurological damages is not an ultimate taboo, and it should be decided considering the risks and advantages by individual conditions of the patients.

When operating regional anesthesia for patients with spinal muscular atrophy, expiratory reserve volume falls if the block area is more than the T6 segment, and the following malfunction of coughing decreases the removing function of the bronchial secretion. In this regard, it is critical to manage the height of the nerve block area properly [14]. By controlling the dose of local anesthetic injected by placing an epidural catheter to operate regional anesthesia, respiratory complications that occur from excessive nervous sense blocks can be prevented, and the anesthesia time can be prolonged for an unexpected extension of the operation. Also, for some patients with spinal muscular atrophy who have accompanying spinal malformation or who had corrected spinal malformation by operating surgeries, the available space for regional anesthesia should be confirmed by a simple radiograph before operation. Technical difficulties can be discovered when operating regional anesthesia for patients who are suffering from scoliosis or had corrected it through surgeries, and, in this case, regional anesthesia can be operated by identifying intervertebral foramen by using C-arm or ultrasonography, or through the spinal lumbarsacral region or scaral foramen. Since the volume of cerebrospinal fluid is decreased for extreme scoliokyphosis, even a little amount of local anesthetia can block sensory nerve up to a higher segment. Accordingly, physicians should pay special attention.

In this case study, the patient complained of paralysis at the intercostal muscle and mild dyspnea. From arterial blood gas analysis, arterial oxygen pressure showed $74.6 \mathrm{mmHg}$, a little lower figure. Although it was not serious, the patient showed gradual paralysis at the sub-respiratory root. Therefore, it was considered that there is the risk of delayed recovery of respiratory function or respiratory complications from difficulties of expectorating bronchial secretion or sputum after general anesthesia. Against this backdrop, regional anesthesia 
was considered to be more beneficial, so operating epidural anesthesia was decided. There were no special opinions on onset time and intensity compared to normal people while clinical duration was increased to 12 hours. However, the increased clinical duration was within the range of the normal upper limit. The height of sensory nerve block of epidural anesthesia is controlled by the dural surface area, while the height of motor nerve block and clinical duration is controlled by posterior epidural fat volume [15]. The cause for extension of clinical duration for epidural anesthesia up to the normal upper limit in this case study is considered to be other factors than spinal muscular atrophy. Consequently, by operating epidural anesthesia to the patient expecting knee synovectomy, bronchial intubation by using skeleton muscle relaxants could be prevented, and since the athletic ability was recovered as good as before the operation, even after removing the epidural catheter, regional anesthesia was conducted without any neurological injuries. Accordingly, the authors consider epidural anesthesia as a good alternative for patients with spinal muscular atrophy expecting a limb surgery.

\section{References}

1. Tsao B, Stojic A. Spinal muscular atrophy. Available from http:// www.emedicine.com/neuro/topic631.htm. Article last updated: Jan $14,2009$.

2. Lefebvre S, Burglen L, Reboullet S, Clermont O, Burlet P, Viollet L, et al. Identification and characterization of a spinal muscular atrophydetermining gene. Cell 1995; 80: 155-65.

3. Sung KH, Lee JM, Lee R. A case of spinal muscular atrophy with extensive involvement of sensory nerves. J Korean Child Neurol Soc
2006; 14: 322-7.

4. Pearn J. Classification of spinal muscular atrophies. Lancet 1980; 1: 919-22.

5. Baum VC, O'Fleisher JE. Anesthesia for genetic, metabolic and dysmorphic syndrome of childhood. 2nd ed. Philadelphia, Lippincott Williams \& Wilkins, a Wolters Kluwer business. 2007, p 206.

6. Upton AR, McComas AJ. The double crush in nerve entrapment syndromes. Lancet 1973; 2: 359-62.

7. Hebl JR, Horlocker TT, Schroeder DR. Neuraxial anesthesia and analgesia in patients with preexisting central nervous system disorders. Anesth Analg 2006; 103: 223-8.

8. Warren TM, Datta S, Ostheimer GW. Lumbar epidural anesthesia in a patient with multiple sclerosis. Anesth Analg 1982; 61: 1022-3.

9. Weston LA, DiFazio CA. Labor analgesia and anesthesia in patient with spinal muscular atrophy and vocal cord paralysis. Reg Anesth 1996; 21 : 350-4.

10. Buettner AU. Anaesthesia for caesarean section in a patient with spinal muscular atrophy. Anaesth Intensive Care. 2003; 31: 92-4.

11. Hobaika AB, Nerves BS. Combined spinal-epidural block in a patient with amyotrophic lateral sclerosis: case report. Rev Bras Anestesiol 2009; 59: 206-9.

12. Choi YH, Gwak M, Lee YK, Lee YM, Lee DH. Intravenous anesthesia in spinal muscular atrophy. Korean J Anesthesiol 2007; 53: 803-5.

13. Hong SJ, Lee JY, Kim DS. General anesthesia with endotracheal intubation for the patient with spinal muscular atrophy. Korean J Anesthesiol 2009; 57: 395-7.

14. Kurup V. Respiratory disease. In: Anesthesia and CO-Existing Disease. 5th ed. Edited by Hines RI, Marschall KE: Philadelphia, Churchill Livingstone. 2008, pp 161-97.

15. Higuchi H, Adachi Y, Kazama T. Factors affecting the spread and duration of epidural anesthesia with ropivacaine. Anesthesiology 2004; 101: 451-60. 